NASA/TP-2001-210195

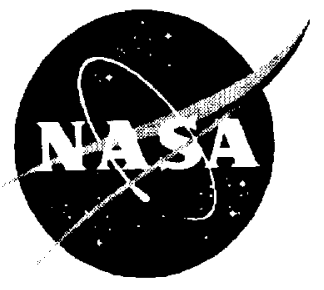

\title{
A Spectral Algorithm for Solving the Relativistic Vlasov-Maxwell Equations
}

John V. Shebalin

Lyndon B. Johnson Space Center

Houston, Texas 77058-3696 


\section{THE NASA STI PROGRAM OFFICE ... IN PROFILE}

Since its founding, NASA has been dedicated to the advancement of aeronautics and space science. The NASA Scientific and Technical Information (STI) Program Office plays a key part in helping NASA maintain this important role.

The NASA STI Program Office is operated by Langley Research Center, the lead center for NASA's scientific and technical information. The NASA STI Program Office provides access to the NASA STI Database, the largest collection of aeronautical and space science STI in the world. The Program Office is also NASA's institutional mechanism for disseminating the results of its research and development activities. These results are published by NASA in the NASA STI Report Series, which includes the following report types:

- TECHNICAL PUBLICATION. Reports of completed research or a major significant phase of research that present the results of NASA programs and include extensive data or theoretical analysis. Includes compilations of significant scientific and technical data and information deemed to be of continuing reference value. NASA's counterpart of peer-reviewed formal professional papers but has less stringent limitations on manuscript length and extent of graphic presentations.

- TECHNICAL MEMORANDUM. Scientific and technical findings that are preliminary or of specialized interest, e.g., quick release reports, working papers, and bibliographies that contain minimal annotation. Does not contain extensive analysis.

- CONTRACTOR REPORT. Scientific and technical findings by NASA-sponsored contractors and grantees.
- CONFERENCE PUBLICATION. Collected papers from scientific and technical conferences, symposia, seminars, or other meetings sponsored or cosponsored by NASA.

- SPECIAL PUBLICATION. Scientific, technical, or historical information from NASA programs, projects, and mission, often concerned with subjects having substantial public interest.

- TECHNICAL TRANSLATION.

English-language translations of foreign scientific and technical material pertinent to NASA's mission.

Specialized services that complement the STI Program Office's diverse offerings include creating custom thesauri, building customized databases, organizing and publishing research results ... even providing videos.

For more information about the NASA STI Program Office, see the following:

- Access the NASA STI Program Home Page at http://www.sti.nasa.gov

- E-mail your question via the Internet to help@sti.nasa.gov

- Fax your question to the NASA Access Help Desk at (301) 621-0134

- Telephone the NASA Access Help Desk at (301) $621-(0390$

- Write to:

NASA Access Help Desk

NASA Center for AeroSpace Information 800 Elkridge Landing Road

Linthicum Heights, MD 21090)-2934 
NASA/TP—2001-210195

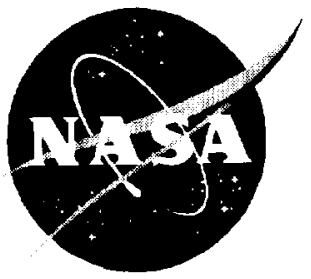

\section{A Spectral Algorithm for Solving the Relativistic Vlasov-Maxwell Equations}

John V. Shebalin

Lyndon B. Johnson Space Center

Houston. Texas 77058-3696

National Aeronautics and

Space Administration

Johnson Space Center

Houston, Texas 77058

January 2001 
Available from:

NASA Center for AeroSpace Information

National Technical Information Service

800 Elkridge Landing Road

5285 Port Royal Road

Linthicum Heights, MD 21090-2934

Springfield, VA 22161

This report is also available in electronic form at http://techreports.larc.nasa.gov/cgi-bin/NTRS 


\section{CONTENTS}

Page

1. The Relativistic Vlasov-Maxwell Equations..........................................................

2. Nondimensional Form of the Equations................................................................. 2

3. Formulation in Velocity Space ............................................................................

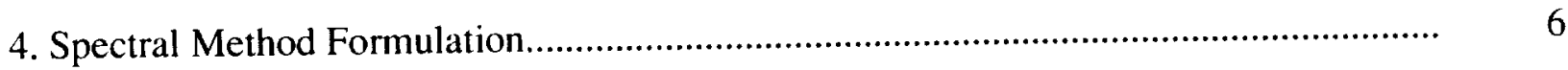

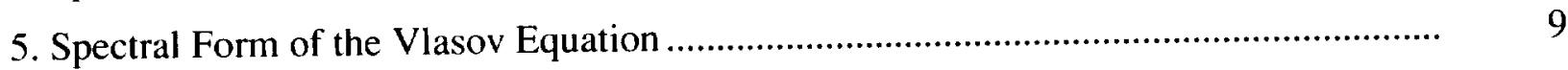

6. Determination of the Electromagnetic Field .......................................................... 12

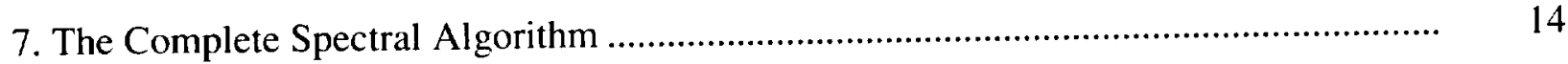

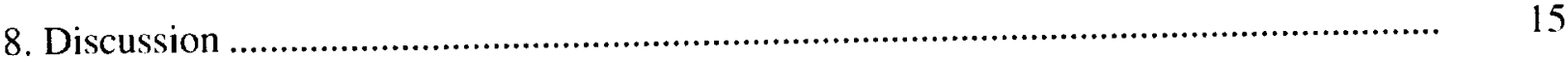

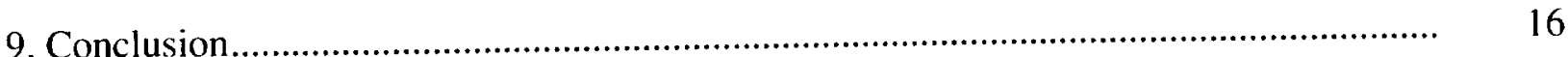

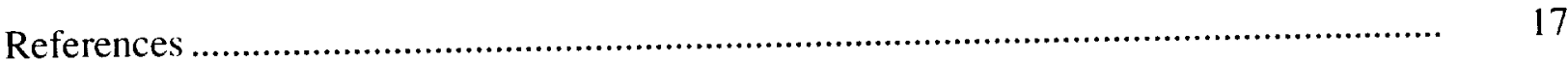




\begin{abstract}
A spectral method algorithm is developed for the numerical solution of the full six-dimensional Vlasov-Maxwell system of equations. Here, the focus is on the electron distribution function, with positive ions providing a constant background. The algorithm consists of a Jacobi polynomial-spherical harmonic formulation in velocity space and a trigonometric formulation in position space. A transform procedure is used to evaluate nonlinear terms. The algorithm is suitable for performing moderate resolution simulations on currently available supercomputers for both scientific and engineering applications.
\end{abstract}




\section{The Relativistic Vlasov-Maxwell Equations}

The dynamics of a high-energy, collisionless plasma are described by the relativistic VlasovMaxwell equations [1]. These nonlinear equations include special relativistic effects [2] and couple the equations of the electromagnetic field (Maxwell's equations) with the evolution equations for single-particle distribution functions (Vlasov equations). In the simplest case, a plasma has two species, protons and electrons. Protons have charge $e>0$, mass $m_{p}$, and distribution function $f_{p}$, while electrons have charge $-e<0$, mass: $m_{\iota}$, and distribution function $f_{\mathfrak{c}}$. Although non-relativistic Vlasov-Poisson and Vlasov-Maxwell systems have received much attention in the distant [3-15] and more recent [16-23] past, relativistic systems appear to be somewhat less explored, although there have been linear treatments [24-27]. Here, an algorithm for a spectral method numerical solution of a fully relativistic, nonlinear Vlasov-Maxwell system is developed. The goal is to set up the basic framework necessary for numerical simulation in the full six-dimensional case. The purpose of this work is to provide a means to enable the study — and ultimately the prediction — of high-energy charged-particle distributions in space. both for intrinsic scientific interest and for optimizing human and robotic space exploration.

The Vlasov equations for the distribution functions $f_{p},(\mathbf{x}, \mathbf{p}, t)$ and $f_{e}(\mathbf{x}, \mathbf{p}, t)$ are

$$
\begin{aligned}
& \frac{\partial f_{p}}{\partial t}+\mathbf{v} \cdot \frac{\partial f_{p}}{\partial \mathbf{x}}+e\left(\mathbf{E}+\frac{\mathbf{v}}{c} \times \mathbf{B}\right) \cdot \frac{\partial f_{p}}{\partial \mathbf{p}}=0 \\
& \frac{\partial f_{e}}{\partial t}+\mathbf{v} \cdot \frac{\partial f_{e}}{\partial \mathbf{x}}-e\left(\mathbf{E}+\frac{\mathbf{v}}{c} \times \mathbf{B}\right) \cdot \frac{\partial f_{e}}{\partial \mathbf{p}}=0
\end{aligned}
$$

To determine the self-consistent fields $\mathbf{E}$ and $\mathbf{B}$, the Maxwell equations are needed:

$$
\text { (1.3) } \nabla \cdot \mathbf{B}=0
$$

$$
\nabla \cdot \mathbf{E}=4 \pi \rho
$$

(1.5) $\frac{1}{c} \frac{\partial \mathbf{B}}{\partial t}=-\nabla \times \mathbf{E}$

(1.6) $\frac{1}{c} \frac{\partial \mathbf{E}}{\partial t}=\nabla \times \mathbf{B}-\frac{4 \pi}{c} \mathbf{j}$. 
The sources present in (1.4) and (1.6) are the charge density $\rho$ and the current density j:

(1.7) $\rho=\rho_{p}+\rho_{e}=e \int\left(f_{p}-f_{e}\right) d \mathbf{p}$

(1.8) $\mathbf{j}=\mathbf{j}_{p}+\mathbf{j}_{\ell}=e \int\left(f_{p}-f_{e}\right) \mathbf{v} d \mathbf{p}$

Additionally, $\mathbf{E}$ and $\mathbf{B}$ can have external components, as well as self-consistent ones, which can serve as external drivers of the coupled Vlasov-Maxwell system.

The solution of this set of integro-differential equations presents a great challenge, because of both the nonlinearity of the couplings and the six-dimensional nature of the solution space of the distribution function. Except in special cases, nonlinearity requires the use of computer simulation, while the presence of six dimensions has, in the past, pushed such simulations beyond the capabilities of then-available computer systems. However, we have now begun to move into an era of fast computers with large core memories, and these machines are providing the resources necessary to perform simulations of six-dimensional continua with a moderate amount of resolution. It is in this context that the following algorithm is presented and we hope that this will help further the development of computer simulations of the Vlasov-Maxwell system and will lead to a greater understanding of the evolution and distribution of high-energy charged particles in space (and other) plasmas.

\section{Nondimensional Form of the Equations}

First, let us nondimensionalize the equations of the previous section. To do this, we denote a characteristic length by $L$, and define

(2.1) $t^{\prime}=\frac{c t}{L}, \quad \mathbf{x}^{\prime}=\frac{\mathbf{x}}{L}, \quad \mathbf{v}^{\prime}=\frac{\mathbf{v}}{c}, \quad \mathbf{p}^{\prime}=\frac{\mathbf{p}}{m c}, \quad \nabla^{\prime}=L \nabla$.

In the previous section, Maxwell's equations were written in Gaussian form, in which the electric field $\mathbf{E}$ and magnetic induction $\mathbf{B}$ have the same units. To nondimensionalize Maxwell's equations, we choose $B_{0}$ as a characteristic electromagnetic field strength and $n_{\mathrm{o}}$ as a characteristic number density, so that the fields and sources are transformed into

(2.2) $\quad \mathbf{E}^{\prime}=\frac{\mathbf{E}}{B_{0}}, \quad \mathbf{B}^{\prime}=\frac{\mathbf{B}}{B_{0}}, \quad \rho_{+}=\frac{\rho_{p}}{e n_{\mathrm{o}}}, \quad \rho_{-}=\frac{\rho_{e}}{e n_{\mathrm{o}}}, \quad \mathbf{j}_{+}=\frac{\mathbf{j}_{p}}{e n_{\mathrm{o}} \mathrm{c}}, \quad \mathbf{j}_{-}=\frac{\mathbf{j}_{\mathcal{C}}}{e n_{\mathrm{o}} c}$ 
Here, the dimensional and dimensionless quantities are functions of $(\mathbf{x}, \mathbf{p}, t)$ and $\left(\mathbf{x}^{\prime}, \mathbf{p}^{\prime}, t^{\prime}\right)$, respectively. Also, using (1.4) and (1.6), a natural choice for $B_{0}$ is $B_{0}=e L n_{0}$, so we will adopt this definition. The nondimensional Maxwell's equations are

$$
\begin{aligned}
& \nabla^{\prime} \cdot \mathbf{B}^{\prime}=0 \\
& \nabla^{\prime} \cdot \mathbf{E}^{\prime}=4 \pi\left(\rho_{+}+\rho_{-}\right) \\
& \frac{\partial \mathbf{B}^{\prime}}{\partial t^{\prime}}=-\nabla^{\prime} \times \mathbf{E}^{\prime} \\
& \frac{\partial \mathbf{E}^{\prime}}{\partial t^{\prime}}=\nabla^{\prime} \times \mathbf{B}^{\prime}-4 \pi\left(\mathbf{j}_{+}+\mathbf{j}_{-}\right)
\end{aligned}
$$

The distribution functions, in turn, take the form:

$$
f_{+}\left(\mathbf{x}^{\prime}, \mathbf{p}^{\prime}, t^{\prime}\right)=\frac{(m c)^{3}}{n_{\mathrm{o}}} f_{p}(\mathbf{x}, \mathbf{p}, t), \quad f_{-}\left(\mathbf{x}^{\prime}, \mathbf{p}^{\prime}, t^{\prime}\right)=\frac{(m c)^{3}}{n_{\mathrm{o}}} f_{e}(\mathbf{x}, \mathbf{p}, t)
$$

The nondimensional Vlasov equation is

$$
\frac{\partial f_{ \pm}}{\partial t^{\prime}}+\mathbf{v}^{\prime} \cdot \frac{\partial f_{ \pm}}{\partial \mathbf{x}^{\prime}} \pm \beta_{ \pm}\left(\mathbf{E}^{\prime}+\mathbf{v}^{\prime} \times \mathbf{B}^{\prime}\right) \cdot \frac{\partial f_{ \pm}}{\partial \mathbf{p}^{\prime}}=0
$$

The constants $\beta_{ \pm}$can be given in terms of $L, n_{0}$, and the classical radius of the electron $r_{\ell}$ :

$$
\beta_{-}=n_{\mathrm{o}} r_{e} L^{2}, \quad \beta_{+}=\frac{m_{e}}{m_{p}} \beta_{-}, \quad r_{e}=\frac{e^{2}}{m_{e} c^{2}}
$$

Unless a different characteristic length $L$ is defined for protons and electrons, the relations in (2.9) indicate that the dynamic coupling of the electromagnetic field to the proton distribution function $f_{+}$is less than that of the field to the electron distribution function $f$ - by a factor of $m_{\mathrm{p}} / m_{\mathrm{e}}$ $=1830$. Assuming that there is only one overall characteristic length $L$, then the dynamic evolution of $f$. can be thought of as occurring on a static proton background, whose sole purpose is to provide overall charge neutrality, at least for times which are short compared to those required for an appreciable evolution of $f_{+}$. Here, it is the electron distribution function evolution that will be of primary concern and, to this end, we will simplify notation by choosing $\beta=1$ and redefining $f_{-}=f$. Furthermore, we will henceforth drop all primes and accept that all variables 
occurring in all equations are dimensionless. The nondimensional equations we will be concerned with are the following:

(2.10) $\frac{\partial f}{\partial t}+\mathbf{v} \cdot \frac{\partial f}{\partial \mathbf{x}}-(\mathbf{E}+\mathbf{v} \times \mathbf{B}) \cdot \frac{\partial f}{\partial \mathbf{p}}=0$

(2.11) $\nabla \cdot \mathbf{B}=0$

(2.12) $\nabla \cdot \mathbf{E}=4 \pi \rho$

(2.13) $\frac{\partial \mathbf{B}}{\partial t}=-\nabla \times \mathbf{E}$

(2.14) $\frac{\partial \mathbf{E}}{\partial t}=\nabla \times \mathbf{B}-4 \pi \mathbf{j}$

(2.15) $p=1-\int f d \mathbf{p}$

(2.16) $\mathbf{j}=-\int f \mathbf{v} d \mathbf{p}$.

\section{Formulation in Velocity Space}

The distribution function depends on $\mathbf{p}$ rather than $\mathbf{v}$ because the six-dimensional phase space volume element $\mathrm{d} \mathbf{x d} \mathbf{p}=d x d y d z d p_{x} d p_{y} d p_{z}$ and the distribution function $f(\mathbf{x}, \mathbf{p})$ are invariant [2] under Lorentz transformations, while $\mathrm{d} \mathbf{x d} \mathbf{v}$ and $f(\mathbf{x , v})$ are not. However, limits on the velocity components are $-1 \leq v_{k} \leq 1$, while the limits on momentum are $-\infty<p_{k}<\infty$. For computational purposes, we will work in velocity space, since the associated finite domain is more commensurate with the finite numerical structure of a digital computer.

The dimensionless relation between momentum and velocity is

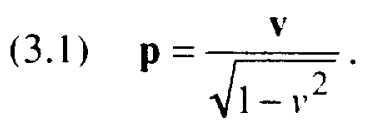

To transform from a momentum space formulation to one in velocity space, we need to calculate the Jacobian of the transformation, and to apply the chain rule of differential calculus to the equations of the previous section. These activities require the following partial derivatives, which can be derived from (3.1): 


$$
\begin{aligned}
& \frac{\partial p_{i}}{\partial v_{k}}=\frac{\left\lfloor\delta_{i k}\left(1-v^{2}\right)+v_{i} v_{k}\right\rfloor}{\left(1-v^{2}\right)^{3 / 2}} \\
& \frac{\partial v_{i}}{\partial p_{k}}=\left(\delta_{i k}-v_{i} v_{k}\right)\left(1-v^{2}\right)^{1 / 2}
\end{aligned}
$$

Here, $d$ is the dimension of the velocity space, and repeated indices, when used, will denote an implicit summation from 1 to $d$ :

(3.4) $v^{2}=v_{i} v_{i} \equiv \sum_{i=1}^{d} v_{i}^{2}$.

The Jacobian for transforming the integrals in (2.15) and (2.16) is

$$
\operatorname{det}\left|\frac{\partial p_{i}}{\partial v_{k}}\right|=\left(1-v^{2}\right)^{-d / 2-1}
$$

Let us also transform the Vlasov equation (2.10) by using (3.2), along with

$$
g(x, v)=f(x, p)
$$

Then, equation $(2.10)$ becomes

$$
\frac{\partial g}{\partial t}+\mathbf{v} \cdot \frac{\partial g}{\partial \mathbf{x}}-(\mathbf{E}+\mathbf{u} \times \mathbf{B})_{i}\left(\boldsymbol{\delta}_{i k}-v_{i} v_{k}\right)\left(1-v^{2}\right)^{1 / 2} \frac{\partial g}{\partial v_{k}}=0 .
$$

(Again, repeated indices denote summation.)

Since no massive particles have a speed of $c$ or greater, we must have $g=0$ for $v^{2} \geq 1$. The distribution function $g$ can be written as

$$
g(\mathbf{x}, \mathbf{v})=\left\{\begin{array}{c}
\left(1-v^{2}\right)^{n} G(\mathbf{x}, \mathbf{v}), \quad v^{2} \leq 1, \quad n>0 \\
0, \quad v^{2}>1
\end{array}\right.
$$

We will assume that $G$ can be represented by an analytic function in the $v_{k}$ over the domain $v^{2} \leq 1$. Also, using $n>1$ in (3.8) will ensure that not only the distribution function, but also its first derivative, will be zero at $v^{2}=1$. 
Using (3.5) and (3.8), the density integral (2.15) becomes

$$
\rho=1-\int_{-\infty}^{\infty} f d \mathbf{p}=1-\int_{-1}^{1}\left(1-v^{2}\right)^{n-\frac{d}{2}-1} G H\left(1-v^{2}\right) d \mathbf{v}
$$

Here, $H(z)$ is the step function, which is equal to unity if $z \geq 0$, and equal to zero otherwise. The current integral (2.16) similarly becomes

(3.10) $\mathbf{j}=-\int_{-\infty}^{\infty} f \mathbf{v} d \mathbf{p}=-\int_{-1}^{1} \mathbf{v}\left(1-v^{2}\right)^{n-\frac{d}{2}-1} G H\left(1-v^{2}\right) d \mathbf{v}$

Using (3.9), equation (3.8) becomes

$$
\frac{\partial G}{\partial t}+\mathbf{v} \cdot \frac{\partial G}{\partial \mathbf{x}}-(\mathbf{E}+\mathbf{u} \times \mathbf{B})_{i}\left(1-v^{2}\right)^{1 / 2}\left[\left(\boldsymbol{\delta}_{i k}-v_{i} v_{k}\right) \frac{\partial G}{\partial v_{k}}-2 n v_{i} G\right]=0
$$

The choice of $n$ in (3.9), (3.10) and (3.11) is determined so as to simplify the algorithm.

\section{Spectral Method Formulation}

Since we wish to consider a fully three-dimensional velocity space, we will set $d=3$. When this three-dimensional velocity space ("v-space") is coupled with a three-dimensional position space, the result is the full six-dimensional phase space of the single particle distribution function. In a three-dimensional relativistic velocity space, the domain is the interior of the sphere $v^{2}=1$, so that a spherical polar coordinate system in velocity space is appropriate:

(4.1) $\quad \mathbf{v}=v[\sin \theta(\cos \varphi \hat{\mathbf{x}}+\sin \varphi \hat{\mathbf{y}})+\cos \theta \hat{\mathbf{z}}]$

Here, $\hat{\mathbf{x}}, \hat{\mathbf{y}}$ and $\hat{\mathbf{z}}$ are orthonormal Cartesian unit vectors. In the velocity-space spherical coordinate system $(1, \theta, \varphi)$, equation (3.11) takes the form:

$$
\frac{\partial G}{\partial t}+\mathbf{v} \cdot \frac{\partial G}{\partial \mathbf{x}}-\left(1-v^{2}\right)^{1 / 2}\left\{(\mathbf{E}+\mathbf{v} \times \mathbf{B}) \cdot \frac{\partial G}{\partial v}-\mathbf{E} \cdot \mathbf{v}\left[v \frac{\partial G}{\partial v}+2 n\left(1-v^{2}\right) G\right]\right\}=0
$$

The density integral (3.10) and corresponding current integral become (here, the element of solid angle is $d \Omega=\sin \theta d \theta d \varphi$ )

$$
\rho=1-\int\left(1-v^{2}\right)^{n-\frac{3}{2}-1} G d \mathbf{v}=1-\int v^{2}\left(1-v^{2}\right)^{n-\frac{3}{2}-1} G d v d \Omega
$$




$$
\mathbf{j}=-\int\left(1-v^{2}\right)^{n-\frac{3}{2}-1} \mathbf{v} G d \mathbf{v}=-\int \mathbf{v} v^{2}\left(1-v^{2}\right)^{n-\frac{3}{2}-1} G d v d \Omega
$$

Our ultimate goal is to simulate numerically the evolution of the distribution function. To this end, a spectral method algorithm will be developed in which dependent variables are expanded in terms of known functions. Since the domain in $\mathbf{v}$-space is a three-dimensional sphere, an appropriate choice of expansion functions for the angular dependence of the $G$ is the well-known spherical harmonics $Y_{n m}(\theta, \varphi)$ [28] (the $P_{n}{ }^{\prime \prime}$ are the associated Legendre functions):

$$
Y_{n m}(\theta, \varphi) \equiv(-1)^{m} \sqrt{\frac{2 n+1}{4 \pi} \frac{(n-m) !}{(n+m) !}} P_{n}^{m}(\cos \theta) e^{i m \varphi} .
$$

A choice of radial expansion functions is complicated by the relativistic factor $\left(1-1^{2}\right)^{n-3 / 2-1}$ in the integrands of (4.3) and (4.4). This difficulty is eliminated by choosing $n=5 / 2$, which removes the relativistic factor from (4.3) and (4.4), although not from (4.2). [If $n=2$ is chosen, the integrands in (4.3) and (4.4) appear to gain a weight function appropriate to Chebyshev polynomials. However, the domain of $v$ is $0 \leq v \leq 1$, while the Chebyshev polynomials $T_{n}(z)$ are orthogonal over the interval $-1 \leq z \leq 1$, and a transformation $z=2 v-1$ produces a weight function no longer in accord with the orthogonality properties of the $T_{l l}(z)$.]

Using $n=5 / 2$ reduces (4.3) and (4.4) to simpler forms:

$$
\begin{aligned}
& \rho=1-\int_{v=0}^{1} d v \int_{\theta=0}^{\pi} d \theta \int_{\varphi=0}^{2 \pi} d \varphi G v^{2} \sin \theta \\
& \mathbf{j}=-\int_{v=0}^{1} d v \int_{\theta=0}^{\pi} d \theta \int_{\varphi=0}^{2 \pi} d \varphi G \mathbf{v} v^{2} \sin \theta
\end{aligned}
$$

The choice of radial expansion function is now straightforward. We will use a set of polynomials that are orthogonal over the interval $0 \leq v \leq 1$, with a weighting function of $v^{2}$. These are the shifted Jacobi polynomials of index $(0,2): P_{n}^{(1.2}(2 v-1)$, which are described in detail elsewhere [29]. Here, the necessary results are 
$P_{0}^{0,2}(s)=1$

$P_{1}^{0.2}(s)=2 s-1$

(4.8)

$P_{2}^{0.2}(s)=\frac{15}{4} s^{2}-\frac{5}{2} s-\frac{1}{4}$

$P_{n+1}^{0.2}(s)=\frac{2 n+3}{n+3}\left[\frac{n+2}{n+1} s-\frac{1}{(n+1)^{2}}\right] P_{n}^{0.2}(s)-\frac{n}{n+3}\left(\frac{n+2}{n+1}\right)^{2} P_{n-1}^{0,2}(s)$

$$
\int_{-1}^{1}(1+s)^{2} P_{n}^{0.2}(s) P_{m}^{0.2}(s) d s=\frac{8}{2 n+3} \delta_{n m}
$$

$$
P_{n+1}^{(0,2}(s)=\frac{(2 n+3)(n+2)}{(n+1)(n+3)}\left[\frac{P_{n}^{0,2}(s)}{n+2}+\frac{n P_{n-1}^{0,2}(s)}{2 n+3}+(n+1) P_{n}^{0,2}(s)\right]
$$

The symbol $P_{n}^{\prime} \equiv d P_{n} / d s$ denotes the first derivative of the $n^{\text {th }}$ order polynomial $P_{n}(s)$.

Using (4.9), let us define the normalized polynomial $R_{n}(v)$ :

$$
\text { a) } R_{n}(v) \equiv \sqrt{2 n+3} P_{n}^{0,2}(2 v-1)
$$

$$
\text { b) } \int_{0}^{1} v^{2} R_{n}(v) R_{m}(v) d v=\delta_{n m}
$$

The distribution function $G$ then has the expansion:

(4.12) $G(v, \theta, \varphi)=\sum_{k=0}^{\infty} \sum_{n=0}^{\infty} \sum_{m=-n}^{n} \gamma_{k n m} R_{k}(v) Y_{n m}(\theta, \varphi)$

Here, it should be noted that the $\gamma_{k n m}=\gamma_{k m m}(\mathbf{x}, t)$ are functions of position $\mathbf{x}$ and time $t$.

The coefficients $\gamma_{k m m}$ can be determined by the inverse operation $\langle\ldots\rangle_{k n m}$ :

(4.13) $\gamma_{k n m}=\langle G\rangle_{k n m} \equiv \int G(v, \theta, \varphi) R_{k}(v) Y_{n m}^{*}(\theta, \varphi) v^{2} d v d \Omega$.

If (4.12) is placed into (4.6) and (4.13) is used, the result is

(4.14) $\rho=1-\gamma_{(0) 0}$. 
To evaluate (4.7), (4.1) can be written as

(4.15) $\mathbf{v}=\sqrt{\frac{4 \pi}{3}} v\left(Y_{11}^{*} \hat{\mathbf{e}}_{+}+Y_{1,-1}^{*} \hat{\mathbf{e}}_{-}+Y_{10}^{*} \hat{\mathbf{e}}_{\mathrm{o}}\right)$.

Here, the complex unit vectors are

$$
\hat{\mathbf{e}}_{+}=-\frac{\hat{\mathbf{x}}+i \hat{\mathbf{y}}}{\sqrt{2}}, \quad \hat{\mathbf{e}}_{-}=\frac{\hat{\mathbf{x}}-i \hat{\mathbf{y}}}{\sqrt{2}}, \quad \hat{\mathbf{e}}_{\mathrm{o}}=\hat{\mathbf{z}} .
$$

Similarly, using (4.1), (4.12) and (4.13), allows the integral (4.7) to be determined:

(4.17) $\mathbf{j}=\sqrt{\pi} \operatorname{Re}\left[\left(\frac{1}{\sqrt{15}} \gamma_{111}+\gamma_{011}\right) \hat{\mathbf{e}}_{+}\right]-\frac{\sqrt{\pi}}{2}\left(\frac{1}{\sqrt{15}} \gamma_{110}+\gamma_{010}\right) \hat{\mathbf{e}}_{\mathrm{o}}$

It is clear that the choice of expansion functions leads to an easy evaluation of the charge density and current, once the expansion functions of the distribution function are known. When $\rho$ and $\mathbf{j}$ are determined, Maxwell's equations (2.11) to (2.14) can be used to find $\mathbf{E}$ and $\mathbf{B}$. These, in turn, affect the evolution of the $\gamma_{k m m}$ through (4.1). The spectral form of the evolution equations will be described next.

\section{Spectral Form of the Vlasov Equation}

The Vlasov equation (4.2) is, in a three-dimensional velocity space spherical polar coordinate system,

$$
\frac{\partial G}{\partial t}+\mathbf{v} \cdot \frac{\partial G}{\partial \mathbf{x}}-\mathbf{E} \cdot \hat{\mathbf{v}}\left(1-v^{2}\right)^{3 / 2}\left(\frac{\partial G}{\partial v}-5 v G\right)-\left(1-v^{2}\right)^{1 / 2}\left(\mathbf{E} \cdot \frac{\mathbf{D}}{v} G-\mathbf{B} \cdot \hat{\mathbf{v}} \times \mathbf{D} G\right)=0
$$

Here $\mathbf{D}$ is the angular derivative part of the velocity space gradient operator:

$$
\frac{\partial}{\partial \mathbf{v}} \equiv \hat{\mathbf{v}} \frac{\partial}{\partial v}+\frac{1}{v} \mathbf{D}, \quad \mathbf{D} \equiv \hat{\theta} \frac{\partial}{\partial \theta}+\frac{\hat{\varphi}}{\sin \theta} \frac{\partial}{\partial \varphi}
$$

In the above, $\hat{v}, \hat{\theta}, \hat{\varphi}$ are the unit vectors in the $v, \theta, \varphi$ directions, respectively.

Now, we subject equation (5.1) to the inverse operation $\langle\cdots\rangle_{k n m}$ defined in (4.12):

$$
\frac{\partial \gamma_{k n m}}{\partial t}+\nabla \cdot \mathbf{N}_{k n m}^{(0)}=\mathbf{E} \cdot\left[\mathbf{N}_{k n m}^{(1)}+\mathbf{N}_{k n m}^{(2)}\right]-\mathbf{B} \cdot \mathbf{N}_{k m m}^{(3)}
$$


where

(5.4) $\quad \mathbf{N}_{k m m}^{(0)}=\langle\mathbf{v} G\rangle_{k n m}$

$$
\mathbf{N}_{k n m}^{(1)}=\left\langle\hat{\mathbf{v}}\left(1-v^{2}\right)^{3 / 2}\left(\frac{\partial G}{\partial v}-5 v G\right)\right\rangle_{k v m}
$$

$$
\mathbf{N}_{k n m}^{(2)}=\left\langle v^{-1}\left(1-v^{2}\right)^{1 / 2} \mathbf{D} G\right\rangle_{k n m}
$$

$$
\mathbf{N}_{k n m}^{(3)}=\left\langle\left(1-r^{2}\right)^{1 / 2} \hat{\mathbf{v}} \times \mathbf{D} G\right\rangle_{k n m}
$$

At this point, we use the properties [28] of the angular momentum operator, L:

$$
\mathbf{L} \equiv-i \hat{\mathbf{v}} \times \mathbf{D}=\frac{1}{\sqrt{2}}\left(L_{+} \hat{\mathbf{e}}_{-}-L_{-} \hat{\mathbf{e}}_{+}\right)+L_{z} \hat{\mathbf{e}}_{\mathbf{o}}
$$

where

$$
L_{ \pm}= \pm e^{ \pm i \varphi}\left(\frac{\partial}{\partial \theta} \pm i \cot \theta \frac{\partial}{\partial \varphi}\right) \quad L_{z}=-i \frac{\partial}{\partial \varphi}
$$

We will also need

$$
\mathbf{D} \equiv-i \hat{\mathbf{v}} \times \mathbf{L}=\frac{1}{\sqrt{2}} \cos \theta\left(L_{+} \hat{\mathbf{e}}_{-}+L_{-} \hat{\mathbf{e}}_{+}\right)-\hat{\mathbf{e}}_{\mathrm{o}} \sin \theta \frac{\partial}{\partial \theta}
$$

(5.11) $\hat{\mathbf{v}}=\frac{1}{\sqrt{2}} \sin \theta\left(e^{i \varphi} \hat{\mathbf{e}}_{-}-e^{-i \varphi} \hat{\mathbf{e}}_{+}\right)+\cos \theta \hat{\mathbf{e}}_{\mathbf{o}}$.

The operators $\hat{\mathbf{v}}, \mathbf{D}$, and $\mathbf{L}$ act on the spherical harmonics $Y_{n m}(\theta, \varphi)$ to produce linear combinations of other spherical harmonics with possibly raised and/or lowered indices $n$ and/or $m$. The coefficients of these linear expansions can be made more succinct using 


$$
\begin{aligned}
C_{n}^{m} & \equiv \sqrt{\frac{(n-m)(n+m)}{(2 n-1)(2 n+1)}} \\
\text { (5.12) } S_{n}^{m} & \equiv \sqrt{\frac{(n+m)(n+m-1)}{(2 n-1)(2 n+1)}} . \\
T_{n}^{m} & \equiv \sqrt{(n-m)(n+m+1)}
\end{aligned}
$$

Using the tabulated properties [28] of the $Y_{n m}(\theta, \varphi)$, along with the above definitions, produces, after a lengthy derivation, the following representations of $(5.4),(5.5),(5.6)$ and $(5.7)$ :

$$
\mathbf{N}_{k n m}^{(\alpha)}=\sum_{j} w_{k, j}^{(\alpha)} \quad\left[\frac{\hat{\mathbf{e}}_{-}}{\sqrt{2}}\left(S_{n+1}^{-m+1} \gamma_{j, n+1, m-1}-S_{n}^{m} \gamma_{j, n-1, m-1}\right)\right.
$$

$$
\begin{aligned}
&+ \frac{\hat{\mathbf{e}}_{+}}{\sqrt{2}}\left(S_{n+1}^{m+1} \gamma_{j, n+1, m+1}-S_{n}^{-m} \gamma_{j, n-1, m+1}\right) \\
&+\left.\hat{\mathbf{e}}_{\mathrm{o}}\left(C_{n+1}^{m} \gamma_{j, n+1, m}+C_{n}^{m} \gamma_{j, n-1, m}\right)\right], \quad \alpha=0, \text { or } 1 \\
& \mathbf{N}_{k n m}^{(2)}=\sum_{j} w_{k, j}^{(2)} \quad\left[\frac{\hat{\mathbf{e}}_{-}}{\sqrt{2}}\left(\{n+m+1\} S_{n+1}^{-m+1} \gamma_{j, n+1, m-1}+\{n-m\} S_{n}^{m} \gamma_{j, n-1, m-1}\right)\right.
\end{aligned}
$$

$$
+\frac{\hat{\mathbf{e}}_{+}}{\sqrt{2}}\left(\{n-m+1\} S_{n+1}^{m+1} \gamma_{j, n+1, m+1}+\{n+m\} S_{n}^{-m} \gamma_{j, n-1, m+1}\right)
$$

$$
\left.+\hat{\mathbf{e}}_{\mathrm{o}}\left(\{n+2\} C_{n+1}^{m} \gamma_{j, n+1, m}-\{n-1\} C_{n}^{m} \gamma_{j, n-1, m}\right)\right]
$$

$$
\mathbf{N}_{k n m}^{(3)}=\sum_{j} w_{k, j}^{(3)} \quad i\left[\frac{\hat{\mathbf{e}}_{-}}{\sqrt{2}} T_{n}^{m-1} \gamma_{j, n, m-1}-\frac{\hat{\mathbf{e}}_{+}}{\sqrt{2}} T_{n}^{-m-1} \gamma_{j, n, m+1}+\hat{\mathbf{e}}_{\mathrm{o}} m \gamma_{j, n, m}\right]
$$

The terms $w_{k, j}^{(\alpha)}$ above are

(5.16) $w_{k, j}^{(0)}=\int_{0}^{1} v^{3} R_{j} R_{k} d v$

(5.17) $w_{k, j}^{(1)}=\int_{0}^{1} r^{2}\left(1-r^{2}\right)^{3 / 2}\left(R_{j}^{\prime}-5 R_{j}\right) R_{k} d v$ 


$$
w_{k, j}^{(2)}=\int_{0}^{1} v\left(1-v^{2}\right)^{1 / 2} R_{j} R_{k} d v
$$

$$
u_{k, j}^{(3)}=\int_{0}^{1} v^{2}\left(1-v^{2}\right)^{1 / 2} R_{j} R_{k} d v
$$

Using (4.8) and (4.11), the integral in (5.16) is relatively simple to evaluate:

$$
w_{k, j}^{(0)}=\frac{1}{2}\left[Q_{k} \delta_{j, k+1}+W_{k} \delta_{j, k}+Q_{k-1} \delta_{j, k-1}\right]
$$

$$
Q_{k}=\frac{(k+1)(k+3)}{(k+2) \sqrt{(2 k+5)(2 k+3)}}, \quad W_{k}=\frac{k^{2}+3 k+3}{(k+1)(k+2)}
$$

The integrals in (5.17), (5.18) and (5.9) are more tedious to evaluate and will be deferred.

We now have a set of partial differential equations (5.3) in position space ( $\mathbf{x}$-space) that is nonlinearly coupled to the Maxwell equations. The velocity dependent part of the Vlasov equation has thus been transformed into a set of $v$-space spectral equations, i.e., equations for the spectra $\gamma_{k m m}(\mathbf{x}, t)$. This $\mathbf{v}$-space spectral formulation is independent of the numerical method that is used in $\mathbf{x}$-space. In $\mathbf{x}$-space, we are free to choose whatever geometry, coordinate system and numerical method seems most appropriate and realizable. In fully ionized plasma problems, there are no distinct bounding surfaces since these generally preclude the existence of a fully ionized state. We must, of course, make some reasonable assumptions about the behavior of the plasma at the numerical boundary. We could assume that the number density falls to zero at such a boundary, for example, or we could assume that density is periodic at the boundary of the numerical grid that represents $\mathbf{x}$-space. This choice then narrows the choices of numerical method to use in $\mathbf{x}$-space. Here, for illustrative purposes and because it is applicable to so-called homogeneous plasmas, we will choose periodic boundary conditions. This leads to a straightforward spectral formulation in $\mathbf{x}$-space. Thus, our next step is to consider solution of the Maxwell equations in the case of periodic boundary conditions.

\section{Determination of the Electromagnetic Field}

The full set of Maxwell's equations, (2.11) to (2.14), can be written in terms of the potential $\varphi$ and the potential vectors $\mathbf{A}$ and $\mathbf{C}$ as follows [30]: 
(6.1) $\frac{1}{c} \frac{\partial \mathbf{A}}{\partial t}=\mathbf{C}$

(6.2) $\frac{1}{c} \frac{\partial \mathbf{C}}{\partial t}=\nabla^{2} \mathbf{A}+4 \pi \mathbf{j}$

(6.3) $\nabla^{2} \varphi=-4 \pi \rho-\nabla \cdot \mathbf{C}$

(6.4) $\frac{1}{c^{2}} \frac{\partial \varphi}{\partial t}+\nabla \cdot \mathbf{A}=0$

Equation (6.4) is the Lorentz condition [31]. The relations (6.1-4) are a set of coupled, firstorder-in-time, partial differential equations for the electromagnetic potentials, in a form suitable for numerical integration. The associated electric and magnetic fields are

(6.5) $\mathbf{B}=\nabla \times \mathbf{A}$

(6.6) $\mathbf{E}=-\nabla \varphi-\mathbf{C}$.

In the case of periodic boundary conditions, we will expand electromagnetic fields and potentials, as well as the $\gamma_{k n m}$, when necessary, using fast Fourier transforms:
a) $\mathbf{A}(\mathbf{x}, t)=(2 \pi)^{-3 / 2} \sum_{\mathbf{k}} \tilde{\mathbf{A}}(\mathbf{k}, t) e^{\mathrm{ik} \cdot \mathbf{x}}$

b) $\tilde{\mathbf{A}}(\mathbf{k}, t)=(2 \pi)^{-3 / 2} \sum_{\mathbf{x}} \mathbf{A}(\mathbf{x}, t) e^{-i \mathbf{k} \cdot \mathbf{x}}$

There is no time-frequency expansion here because the coupling between the electromagnetic field and matter is generally nonlinear, which generally precludes a simple dispersion relation between wave frequency $\omega$ and wave vector $\mathbf{k}$. In terms of Fourier coefficients (omitting $t$ in the argument, for brevity), equations (6.1) to (6.6) take the form:

(6.8) $\frac{1}{c} \frac{d \tilde{\mathbf{A}}(\mathbf{k})}{d t}=\widetilde{\mathbf{C}}(\mathbf{k})$
(6.9) $\frac{1}{c} \frac{d \widetilde{\mathbf{C}}(\mathbf{k})}{d t}=-k^{2} \widetilde{\mathbf{A}}(\mathbf{k})+4 \pi \tilde{\mathbf{j}}(\mathbf{k})$ 
(6.10) $\tilde{\varphi}(\mathbf{k})=k^{-2}[4 \pi \tilde{\rho}(\mathbf{k})+\mathbf{i k} \cdot \widetilde{\mathbf{C}}(\mathbf{k})]$

(6.11) $\frac{1}{c^{2}} \frac{d \widetilde{\varphi}(\mathbf{k})}{d t}+\mathrm{ik} \cdot \widetilde{\mathbf{A}}(\mathbf{k})=0$.

The sources in (6.9) and (6.11) are the Fourier transforms, using (6.7), of (4.14) and (4.17). (Also, note that the source coefficients corresponding to $\mathbf{k}=0$ are zero.)

Using (6.7) and (6.10), equations (6.5) and (6.6) become

(6.12) $\widetilde{\mathbf{B}}(\mathbf{k})=\mathrm{ik} \times \widetilde{\mathbf{A}}(\mathbf{k})$

(6.13) $\tilde{\mathbf{E}}(\mathbf{k})=-4 \pi i \frac{\mathbf{k}}{k^{2}} \widetilde{\rho}(\mathbf{k})-\left(\mathbf{I}-\frac{\mathbf{k k}}{k^{2}}\right) \widetilde{\mathbf{C}}(\mathbf{k})$.

Here, I is the unit dyadic. Note that $\widetilde{\varphi}(\mathbf{k})$ does not appear in these expression for $\widetilde{\mathbf{B}}(\mathbf{k})$ and $\widetilde{\mathbf{E}}(\mathbf{k})$. The scalar potential is thus only an auxiliary function, while $\widetilde{\mathbf{A}}(\mathbf{k})$ and $\widetilde{\mathbf{C}}(\mathbf{k})$ are seen to be more fundamental in classical electrodynamics. Furthermore, it is only those parts of $\tilde{\mathbf{A}}(\mathbf{k})$ and $\widetilde{\mathbf{C}}(\mathbf{k})$ transverse to $\mathbf{k}$ that are essential to determining $\widetilde{\mathbf{B}}(\mathbf{k})$ and $\widetilde{\mathbf{E}}(\mathbf{k})$. (However, in numerical simulations of quantum electrodynamic processes, $\widetilde{\varphi}(\mathbf{k})$ must also be calculated $[32,33]$.)

\section{The Complete Spectral Algorithm}

A spectral method algorithm for the Vlasov-Maxwell system, as developed here, consists of a part in velocity space and a part in position space. The basic $\mathbf{v}$-space spectral equation is (5.3) and its solution produces the coefficients $\gamma_{k n m}$, which are time-dependent functions in $\mathbf{x}$-space. We use a small subset of these coefficients to directly determine the electromagnetic sources, through (4.14) and (4.17). In $\mathbf{x}$-space, Fourier transforms of these sources then allow the timeevolution of the electric and magnetic fields to be calculated through (6.8), (6.9), (6.12), and (6.13). These electromagnetic fields then enter the right-hand side of (5.3), completing the cycle. This is a coupled set of nonlinear equations, and a complete description of the algorithm developed here requires only a few additional details. 
Equation (5.3) determines $\gamma_{k n m}$ and can be written as

(7.1) $\frac{\partial \gamma_{k n m}}{\partial t}=\Gamma_{k n m}+\Psi_{k n m}$

where

$$
\Gamma_{k n m}=-\nabla \cdot \mathbf{N}_{k n m}^{(0)}, \quad \Psi_{k n m}=\mathbf{E} \cdot\left\lfloor\mathbf{N}_{k n m}^{(1)}+\mathbf{N}_{k n m}^{(2)}\right\rfloor-\mathbf{B} \cdot \mathbf{N}_{k n m}^{(3)}
$$

To determine the $\Gamma_{k m m}$ we use the Fourier transform to k-space $(6.20 \mathrm{~b})$, to find $\tilde{\mathbf{N}}_{k n m}^{(0)}$ and then form

$$
\text { (7.3) } \tilde{\Gamma}_{k n m}=-i \mathbf{k} \cdot \tilde{\mathbf{N}}_{k n m}^{(0)}
$$

after which we transform back to $\mathbf{x}$-space with (6.20a) to get $\Gamma_{k n m}$. Similarly, using (6.20a) to find $\mathbf{E}(\mathbf{x})$ and $\mathbf{B}(\mathbf{x})$ from $\widetilde{\mathbf{E}}(\mathbf{k})$ and $\widetilde{\mathbf{B}}(\mathbf{k})$, allows us to form $\Psi_{k n m}$ point-wise on the numerical $\mathbf{x}$ space grid. The transform method was pioneered many years ago [6] and has proven very useful in the solution of nonlinear problems involving many modes.

Equation (7.1) is now defined on all $\mathbf{x}$-space grid points and can be used to advance the $\gamma_{k n m}$ forward in time with a suitable time-integration scheme (such as a Runge-Kutta [34] or an Adams-Bashforth [35] procedure). Since $\Gamma_{k n m}$ consists of only six coefficients $\gamma_{k m m}$, it may be efficient and more accurate to treat this term implicitly when solving (7.1). Aliasing (i.e., higherorder coefficients affecting the determination of lower-order coefficients) can occur in determining the $\Psi_{k n m}$, but this can be eliminated, if desired [36]. However, aliasing is often not critical, and run time can be reduced by neglecting aliasing effects (such methods are termed pseudospectral [37]). Lastly, one can introduce external parts to $\mathbf{E}$ and $\mathbf{B}$ in (7.2), in addition to those parts determined by (6.12) and (6.13), and these can serve as a source of external forcing, if appropriate to a given problem.

\section{Discussion}

The spectral method algorithm developed here provides a means for solving the coupled, nonlinear Vlasov-Maxwell system of equations in the full six-dimensional case. Numerical implementation requires the use of a discrete grid of points, and if we assume, for the purpose of illustration, that each dimension is given $N$ points, then there are a total of $N^{6}$ grid points. If $N=$ 32 , then $N^{6} \approx 10^{9}$, and if we assume 8 bytes per word in a computer, along with a factor of 25 to 
50 for all the arrays needed, then core memory requirements are in the range of 1 to 2 terabytes ( $10^{12}$ bytes), which is currently available on the largest existing supercomputers. The number of grid points allotted to each dimension in the six-dimensional $x$ - $v$-space can also vary, to increase or decrease resolution, as appropriate.

However, remember that, in a spectral method, grid points are sampling points for an underlying continuous representation. The number of grid points is thus essentially equivalent to the number of coefficients (the 'spectra') kept after truncating a formally infinite function expansion. To prevent a 'pileup' at the higher-order coefficients and to ameliorate the effects of 'filamentation' $[10,12,17]$, it may prove necessary to investigate the use of a collision term in the Vlasov equation, as done previously $[3,16]$. (These possibilities, and others unthought of, remain as potential challenges for future development.)

In spherical polar coordinates in $\mathbf{v}$-space, the number of points in the radial, or $v$, direction sets the energy resolution of the code. This resolution may be defined by the relativistic factor $\gamma_{n}=$ $\left(1-v_{n}{ }^{2}\right)^{-1 / 2}$ corresponding to the largest positive zero $v_{n}$ of $R_{n}(v)$ (all the zeros are used in the Gaussian quadrature formula [38] for integrals involving $R_{n}$, in which case the function to be evaluated is effectively known more or less precisely only at those zeros and interpolated in between [39]). Using known results [29], we can estimate $\gamma_{n} \approx 8 n / 3 \pi$. For $n=32$, this gives the resolution of electron energies an upper bound of around $14 \mathrm{MeV}$, a value which can be increased by making $n$ larger (e.g., by decreasing the number of grid points allotted to other coordinates). Thus, moderate resolution six-dimensional numerical simulations of relativistic electron distributions are possible on available computers.

\section{Conclusion}

The algorithm presented here provides a straightforward procedure with which to begin using supercomputer resources. The next steps are to evaluate certain integrals mentioned in this paper, to begin the numerical implementation of the algorithm, to gain access to the necessary computers, and to begin running numerical simulations. The probable outcome of proceeding is the creation of a numerical tool capable of studying the full six-dimensional dynamic evolution of charged-particle distributions, starting from various initial conditions and taking into account the influence of external fields. The benefits to be gained are a greater understanding of basic plasma processes and a new capability to predict radiation levels and effects in astrophysical, spaceflight, and engineering systems. 


\section{References}

1. E. M. Lifshitz and L. P. Pitaevskii, Physical Kinetics (Pergamon, New York, 1981), pp. 117-8, 205-6.

2. L. D. Landau and E. M. Lifshitz, The Classical Theory of Fields, $4^{\text {th }}$ Ed (Pergamon, New York, 1975), pp. 29-30.

3. T.P. Armstrong and D. Montgomery, Asymptotic state of the two-stream instability, $J$. Plasma Phys. 1, 425-433 (1967).

4. J. Denavit, B. W. Doyle, and R. H. Hirsch, Nonlinear and collisional effects on Landau damping, Phys. Fluids 11, 2241-9 (1968).

5. T. P. Armstrong and D. Montgomery, Numerical study of weakly unstable electron plasma oscillations, Phys. Fluids 12, 2094-8 (1969).

6. T. P. Armstrong, R. C. Harding, G. Knorr, and D. Montgomery, Solution of Vlasov's equation by transform methods, in Methods in Computational Physics, Vol. 9, edited by B. Adler (Academic Press, New York, 1970), pp. 29-86.

7. G. Joyce, G. Knorr, and T. Burns, Nonlinear behavior of the one-dimensional weak beam plasma system, Phys. Fluids 14, 797-801 (1971).

8. G. Joyce and G. Knorr, Numerical integration methods of the Vlasov equation, J. Comput. Phys. 8, 53-63 (1971).

9. J. Denavit and W. L. Kruer, Comparison of numerical solutions of the Vlasov equation and particle simulations of collisionless plasmas, Phys. Fluids 14, 1782-1791 (1971).

10. J. Denavit, Numerical simulation of plasmas with periodic smoothing in phase space, $J$. Comput. Phys. 9, 75-98 (1972).

11. G. Knorr, Plasma simulation with few particles, J. Comput. Phys. 13, 165-180 (1973).

12. C. Z. Cheng and G. Knorr, The integration of the Vlasov equation in configuration space, $J$. Comput. Phys. 22, 330-351 (1976).

13. R. R. J. Gagné and M. M. Shoucri, A splitting scheme for the numerical solution of a onedimensional Vlasov equation, J. Comput. Phys. 24, 445-9 (1977).

14. A. Ghizzo, B. Izrar, P. Bertrand, E. Fijalkow, M. R. Feix, and M. Shoucri, Stability of Berstein-Greene-Kruskal plasma equilibria. Numerical experiments over a long time, Phys. Fluids 31, 72-82 (1988). 
15. J. Denavit, Simulations of the single-mode, bump-on-tail instability, Phys. Fluids 28, 27737 (1988).

16. L. Demeio, The inclusion of collisional effects in the splitting scheme, J. Comput. Phys. 99 , 203-8 (1992).

17. A. Klimas \& W. M. Farrell, A splitting algorithm for Vlasov simulation with filamentation filtration, J. Comput. Phys. 140, 150-163 (1994).

18. J. P. Holloway, Spectral velocity discretizations for the Vlasov-Maxwell equations, Transport Theory and Stat. Phys, 25, 1-32, 1996.

19. J. P. Holloway, On numerical methods for Hamiltonian PDEs and a collocation method for the Vlasov-Maxwell equations, J. Comput. Phys. 129, 121-133 (1996).

20. S. Wollman and E. Ozizmir, Numerical approximation of the one-dimensional VlasovPoisson system with periodic boundary conditions, SIAM J. Numer. Anal. 33, 1377-1409 (1996).

21. J. W. Schumer and J. P. Holloway, Vlasov simulations using velocity-scaled Hermite representations, J. Comput. Phys. 144, 626-66I (1998).

22. T. Utsumi, T. Kunugi, and J. Koga. A numerical method for solving the Vlasov equation in phase space. Comp. Phys. Comm. 108, 159-179 (1998).

23. T. Nakamura and T. Yabe, Cubic interpolated propagation scheme for solving the hyperdimensional Vlasov-Poisson equation in phase space, Comp. Phys. Comm. 120, 122 154 (1999).

24. P. C. Clemmow and A. J. Willson, The dispersion equation in plasma oscillations, Proc. Roy. Soc. A237, 117-131 (1956)

25. B. Buti, Plasma oscillations and Landau damping in a relativistic gas, Phys. Fluids 5, 1-5 (1962).

26. W. B. Thompson, The self-consistent test particle approach to relativistic kinetic theory, in Kinetic Equations, edited by R. Liboff and N. Rostoker (Gordon \& Breach, New York, 1971), pp. 79-89.

27. R. C. Davidson, H. Qin, and P. H. Stolz, Kinetic description of the electron-proton instability in high intensity proton linacs and storage rings based on the Vlasov-Maxwell equations, Phys. Rev. Special Topics - Accelerators and Beams 2, 054401 (1999).

28. G. B. Arfken and H. J. Weber, Mathematical Methods for Physicists, $4^{\text {th }}$ Ed. (Academic Press, New York, 1995), pp. 736-742 \& p. 124. 
29. I. S. Gradshteyn and I. M. Ryzhik, Table of Integrals, Series, and Products (Academic Press, New York, 1965), sec. 8.96.

30. J. V. Shebalin, Electromagnetic potential vectors and spontaneous symmetry breaking, Nuovo Cimento, 108 B, 99-101 (1993).

31. J. D. Jackson, Classical Electrodynamics, $2^{\text {nd }}$ Ed. (Wiley, New York, 1975), pp. 220-3.

32. J. V. Shebalin, Homogeneous quantum electrodynamic turbulence, Physica D, 66, 381-391 (1993).

33. J. V. Shebalin, Numerical solution of the coupled Dirac and Maxwell equations, Physics Letters A, 226, 1-6 (1997).

34. F. B. Hildebrand, Introduction to Numerical Analysis, $2^{\text {nd }}$ Ed. (Dover, New York, 1974), pp. 285-292.

35. J. Gadzag, Time-differencing schemes and transform methods, J. Comput. Phys. 20, 196207 (1976).

36. G. S. Patterson and S. A. Orszag, Spectral calculations of isotropic turbulence: Efficient removal of aliasing interactions, Phys. Fluids, 14, 2358 (1971).

37. B. Fornberg. A Practical Guide to Pseudospectral Methods (Cambridge, UK, 1996).

38. M. Abramowitz and I. A. Stegun, Handhook of Mathematical Functions, AMS 55 (National Bureau of Standards, USGPO, Washington, DC, 1970), p. 888, no. 25.4.33.

39. C. Lanczos, Applied Analysis (Dover, New York, 1988), Chap. VI. 




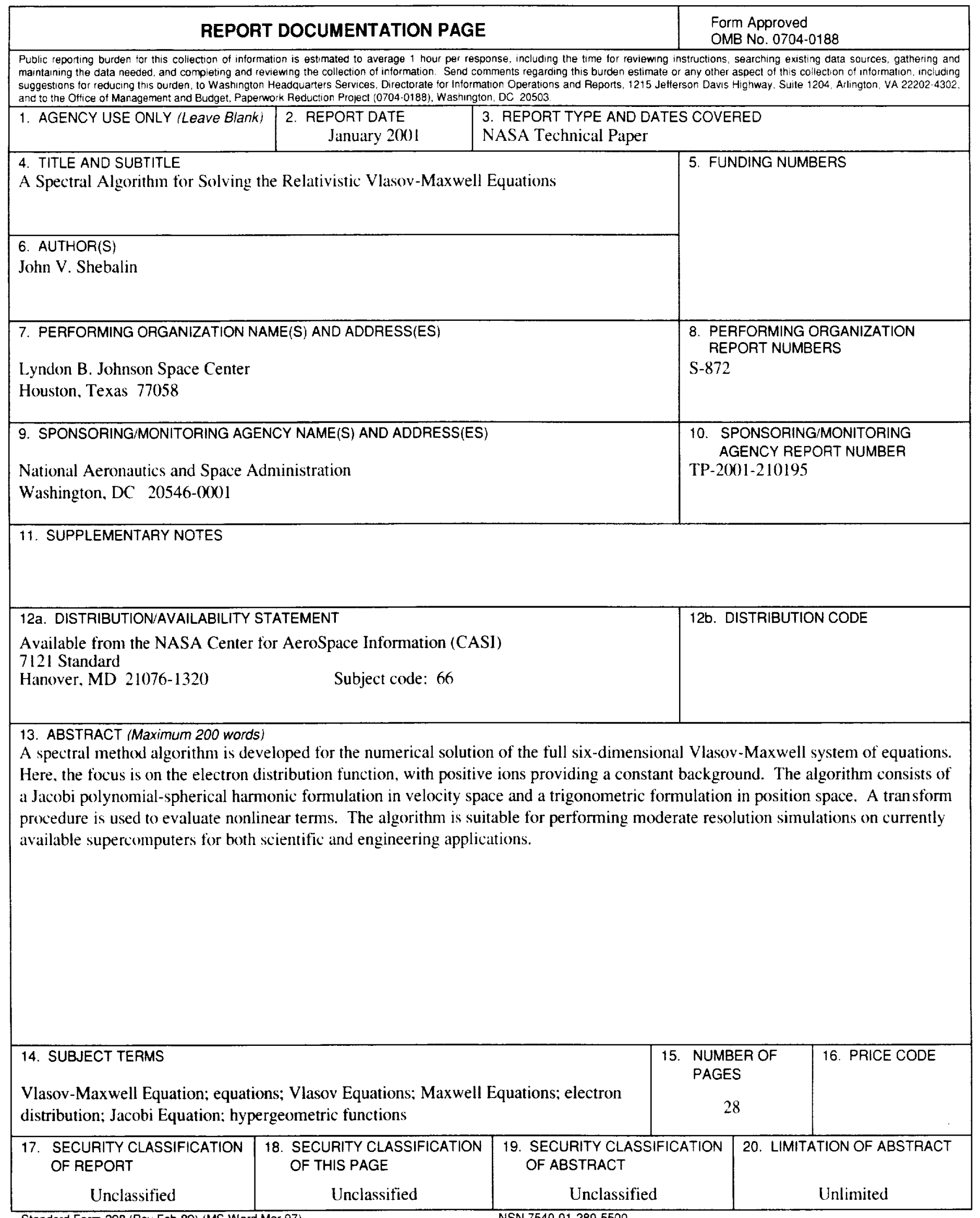

Standard Form 298 (Rev Feb 89) (MS Word Mar 97)

Prescribed by ANSI Std. 239-18

298-102 
